\title{
Glaucoma Diagnosis - The Role of Optic Nerve Examination
}

\author{
a report by \\ Hans G Lemij \\ Scientific Director and Eye Surgeon, Rotterdam Eye Hospital
}

DOI: 10.17925/EOR.2007.00.00.15

Glaucoma is a chronic progressive disease that, left untreated, can lead to blindness. Glaucoma represents a range of conditions in which the flow of aqueous humour in the eye is blocked, causing an increase in intraocular pressure (IOP). An impaired blood flow to the optic disc may also cause glaucoma, even without an abnormally high IOP. Even with careful monitoring and lowering of IOP, approximately $25 \%$ of patients lose their sight. ${ }^{1}$ Consequently, glaucoma is the second most common cause of blindness worldwide.

The global prevalence of glaucoma was estimated to be 67 million in 2001.2 Approximately $13 \%$ of these were estimated to be in Europe, of which half were undiagnosed and untreated. The most prevalent type of glaucoma, primary open-angle glaucoma (POAG), has subtle symptoms early in its course and, consequently, often remains undetected. Late diagnosis of glaucoma significantly increases the cost of treatment and raises the risk of visual impairment. ${ }^{3}$ Historically, an increased level of IOP was presumed to be the most accurate means of diagnosis; however, it is now recognised that high IOP is a risk factor for glaucoma, but is not an accurate diagnostic. Therefore, there is a need for an accurate diagnosis system.

\section{Glaucoma Diagnosis - The 'Gold Standard' Tests}

In glaucoma, structural changes usually occur first and are followed by functional deficits. There are three well-established 'gold standard' tests used by ophthalmologists to diagnose glaucoma: IOP measurements, visual field tests and (stereoscopic) assessments of the optic nerve. Together, these methods provide information on both structural and functional defects.

\section{Intraocular Pressure}

A gradual increase in IOP has long been recognised as the major risk factor for glaucoma, and the lowering of IOP serves to impede progression of optic nerve damage. Therefore, IOP measurements are usually documented over time. ${ }^{4}$ The Goldmann applanation tonometer is the standard device used by physicians to detect alterations in IOP. The device can accurately measure IOP in the eye with a small deviation of $0.5 \mathrm{mmHg} .^{5}$

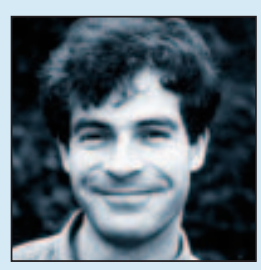

Hans $\mathrm{G}$ Lemij is a glaucoma specialist at the Rotterdam Eye Hospital in Rotterdam, The Netherlands, and a Visiting Professor at the Catholic University of Leuven, Belgium. His primary interests are research and education in glaucoma imaging, structure-function relationships in glaucoma and glaucoma shared care. He has also carried out research in neuro-ophthalmology, lax eyelid syndrome, laser polarimetry and optic nerve imaging.

E: lemij@wxs.n
Although the device is fairly accurate, the measurement of IOP is not always a precise indication of glaucoma. Individually, IOP is highly variable, and even low IOP cannot be ruled out for risk of glaucoma. Additionally, IOP does not indicate the extent of damage or, indeed, that damage has actually been done to the optic nerve. Thus, in the diagnosis of glaucoma this variable can be used only alongside other evidence for a positive outcome.

\section{Perimetry}

Peripheral vision is usually the first to deteriorate in glaucoma; hence, tests of the visual field have been used to diagnose the disease. Perimetry is a systematic measurement of light sensitivity in the visual field by the detection of targets presented on a defined background. The standard diagnostic tool for visual field examination is the computerised field analyser. Responses are statistically analysed and compared with a database of normal responses. Even with this comparison it is challenging to state definitively whether a patient has glaucoma. Previously, for detecting any progression the test results were judged by comparing print-outs from visual field tests, which was time-consuming and often inaccurate. Today, new software provides ophthalmologists with automated visual-field-progression analysis; however, it may take three examinations before an accurate baseline is obtained. Also, long-term fluctuations in the field tests can often occur, thus the accuracy of this method of diagnosis is still in question.

\section{Stereoscopic Optic Nerve Photographs}

Glaucoma leads to alterations in the size and shape of the optic disc, the neuroretinal rim and the size ratio between the optic disc and cup. By means of photographs of the optical nerve head $(\mathrm{ONH})$, morphological changes in glaucoma are qualitatively monitored. In addition, significant thinning of the retinal nerve fibre layer (NFL) demonstrates early-stage glaucoma. ${ }^{6}$ Thus, evaluation of the $\mathrm{ONH}$ is the mainstay of the three components of making a diagnosis. However, the appearance of the ONH differs widely between individuals, which can make it difficult to detect glaucomatous eyes.

Limitations of the Traditional Methods

The standard diagnostic techniques have several limitations, the most important of which is insensitivity. It is generally recognised that IOP measurement is a particularly insensitive and non-specific way of making a diagnosis of glaucoma. Visual field testing is a fairly insensitive method for early diagnosis, and stereoscopic evaluation of the $\mathrm{OHN}$ is very subjective and open to misinterpretation.

A recent study found that the clinical assessment of stereoscopic ONH photographs for diagnosing glaucoma varied greatly and was dependent on the level of training. ${ }^{7}$ In the study, stereoscopic $\mathrm{ONH}$ photographs were obtained from one eye each of 40 healthy subjects, 
48 glaucoma patients and six patients with ocular hypertension. Four glaucoma specialists, four general ophthalmologists, four residents in ophthalmology and four optometrists classified the ONH photographs as normal or glaucomatous. The study found that glaucoma specialists and general ophthalmologists had the greatest diagnostic accuracy, with residents classifying the fewest eyes correctly. The intra- and interobserver agreement for $\mathrm{ONH}$ analysis was only moderate to good.

An ongoing pan-European study is assessing the consistency of stereoscopic $\mathrm{ONH}$ analysis in 300 general ophthalmologists from 11 European countries. The European Optic Disc Assessment Trial (EODAT) has a similar design to the investigation conducted by Reus et al.7 Stereoscopic optic disc photographs of one eye each of 40 healthy subjects, 48 glaucoma patients and six patients with ocular hypertension were assessed by the participating ophthalmologists. Preliminary data from 136 general ophthalmologists from nine European countries showed that the accuracy of European ophthalmologists for classifying optic disc photographs in glaucoma varied considerably. The study even found it to vary between countries. The intraobserver agreement from the preliminary data was moderate to very good. ${ }^{7}$ Worryingly, some participants even approached the $50 \%$ chance level.

\section{Beyond the Current Standard Tests}

Structural assessment is fundamental for early glaucoma diagnosis and in the management of the disease. Alterations of the ONH and the NFL are the first signs of glaucoma, commonly appearing ahead of any functional manifestations. ${ }^{8}$ Newer ONH imaging devices and NFL analysers adopt a different objective strategy from the traditional techniques, which is not dependent on patient responses. These tests measure the physical thickness of the NFL in the retina. In glaucoma the nerve fibres are lost, thus patients with glaucoma are likely to have a thinner NFL than normal. In red-free fundus photographs, NFL damage due to glaucoma results in darker, often wedge-shaped areas. These dark areas are slightly larger than arterioles, reach the disc following the normal course of the NFL and represent retrograde degeneration of the axons due to focal damage of the optic nerve at the lamina. Wedge defects are caused by atrophy of many ganglion cells in the same area of the optic nerve. By measuring changes in NFL thickness over time these tests can detect glaucoma progression, as well as providing additional early information for

\section{It is now recognised that high intraocular pressure is a risk factor for glaucoma, but is not an accurate diagnostic. Therefore, there is a need for an accurate diagnosis system.}

determining whether a patient has glaucoma. Accordingly, these new imaging techniques may assist clinicians in diagnosing glaucoma. Three useful tools have made the transition from the laboratory to the clinic: scanning laser polarimetry (SLP), confocal scanning laser ophthalmoscopy (CSLO) and optical coherence tomography (OCT).

\section{Scanning Laser Polarimetry}

SLP utilises the birefringent properties of the NFL to measure its thickness by measuring the phase-shift of polarised light through the layer. The initial version of the current GDx machine was the first commercially available SLP device. However, the technology initially suffered from various limitations, such as motion artefacts and, more importantly, spurious measurements originating from poorly compensated corneal phase-shifts of the polarised scanning laser beam. ${ }^{9}$

The standard diagnostic techniques

have several limitations, the most important of which is insensitivity.

The new-generation GDx-VCC improves on previous devices, mainly in that it offers variable corneal compensation (VCC). Previously, SLP devices assumed a fixed slow axis of corneal birefringence of $15^{\circ}$ nasally downwards in every eye. Recent studies have shown a large variation of birefringent properties between patients, hence limiting the ability of the older devices to detect glaucoma. 10,11 The updated GDx includes a VCC for customised compensation of individual corneal properties. An increase in diagnostic accuracy was noted in glaucoma detection between the GDx-VCC device and previous devices with a fixed corneal compensation. ${ }^{12}$ The GDx-VCC device has a sensitivity of $84 \%$ for a given specificity of $94 \%$ when examining the NFL in glaucoma patients. ${ }^{13}$ An even greater corneal compensation - so-called enhanced corneal compensation (ECC) - will be released shortly. It has been shown to offer an even greater diagnostic accuracy than VCC. There are still limited data on the improvements offered by the device in glaucoma progression detection, and further studies need to be performed to discover its full potential.

\section{Confocal Scanning Laser Ophthalmoscopy}

The principle of light reflection is employed in CSLO. In this technique a laser is projected through a pinhole towards the area of interest. ${ }^{14}$ The reflections are picked up though another pinhole in front of a light detector. The pinholes ensure that only light reflected from a defined focal plane will reach the detector. A series of scans is undertaken and reconstructed to produce a $3 \mathrm{D}$ image.

The main drawback of CSLO is the generation of topographical parameters using a moderately reproducible reference plane. It is unclear to what extent blood vessels in the ONH affect the measurements. Furthermore, the operator must also outline the disc margin, which is a time-consuming and subjective task. More recent software eliminates the need for operator-dependent margin demarcation. An automated classifier - the glaucoma probability score (GPS) - quite accurately classifies healthy and glaucomatous discs. In the clinic, the most commonly used CSLO device is the Heidelberg retina tomograph (HRT). The device includes an internal fixation target, improving its reproducibility. Glaucomatous progression has been demonstrated with the HRT, although it agreed only moderately with visual field progression. ${ }^{15}$

\section{Optical Coherence Tomography}

OCT, based upon interferometry, is a relatively new technique for optical imaging. It is a non-invasive, non-contact method of producing 
high-resolution images using light reflected from a scanned area. ${ }^{16}$ The technique is analogous to ultrasound and can be used to produce anterior and posterior segment imaging of the retina, as well as to measure the NFL thickness to evaluate the presence of glaucoma. ${ }^{17}$ The OCT technique has been used primarily by retina specialists, and occasionally by glaucoma specialists. However, studies with OCT in glaucoma have demonstrated that it discriminates quite well between healthy and glaucomatous eyes.18,19 Its role in detecting any glaucomatous progression is uncertain. A new-generation OCT frequency-domain OCT (also known as spectral-domain OCT or Fourierdomain OCT) - has recently entered the market. This technology provides high-speed and high-resolution images. It holds great promise for becoming the gold standard of structural imaging of glaucoma, although the published data are still very limited.

\section{The Future 'Gold Standard' in Glaucoma Diagnosis?}

The importance of $\mathrm{ONH}$ and NFL assessment in the diagnosis of glaucoma cannot be understated. The new imaging devices can be used to evaluate patients in whom there is suspected glaucomatous $\mathrm{ONH}$ and NFL damage, but who have yet to show visual field defects on standard

Three useful tools have made the

transition from the laboratory to the

clinic: scanning laser polarimetry,

confocal scanning laser ophthalmoscopy

and optical coherence tomography.

perimetry. Several studies have evaluated these devices in clinical applications. Newer perimetric tests may detect glaucomatous damage earlier than standard visual field tests, although their role is still uncertain.

A recent study compared the accuracy of HRT, GDX-VCC and OCT in the diagnosis of glaucoma, and concluded that there was no significant difference between the diagnostic capabilities for glaucoma between the three devices using the best parameters for each. ${ }^{20}$ Another study examining the devices showed that subjective assessment of the $\mathrm{ONH}$ provided the best diagnostic efficacy for glaucoma. ${ }^{21}$ However, recent studies have shown that automated analysis of measurements with GDxVCC and HRT had a diagnostic accuracy for glaucoma either equal to or higher than the classification of stereoscopic ONH photographs by glaucoma specialists. ${ }^{?}$

It is also important when following up patients that the ONH and NFL be well documented. The beauty of the recent imaging devices is that they provide an objective means of documenting glaucoma, facilitating the

The beauty of the recent imaging

devices is that they provide an

objective means of documenting

glaucoma, facilitating the detection

of any progression.

detection of any progression. In the past photographs were used, but these have their limitations. Frequency-domain OCT may well become the gold standard for imaging glaucoma, for both its detection and the follow-up of any progression.

\section{Summary and Conclusions}

Glaucoma is a chronic progressive disease in which approximately $50 \%$ of sufferers are undiagnosed. The early stages of the disease show structural changes to the $\mathrm{ONH}$ and NFL, and are difficult to detect using current visual field tests or IOP measurements. Judging the $\mathrm{ONH}$ is currently the most common test for early diagnosis of glaucoma. However, these old mainstays are quite inadequate and, in addition, they provide very little documentation for follow-up. Promising devices such as SLP, CSLO and OCT have proved to give comparable reproducibility and accuracy in the evaluation of glaucomatous and normal eyes, and may assist clinicians in diagnosing glaucoma. Their role in detecting progression is currently being explored by several research groups. The latest-generation OCT holds great promise for both detecting and following glaucoma, although its future role is still unclear. Moreover, there is a need to improve education and training in examining the $\mathrm{ONH}_{\text {; }}$ in turn, this will improve earlier glaucoma detection and thus treatment.
1. Leydhecker W, Graner E, Long-term studies of visual field changes by means of computerised perimetry in eyes with glaucomatous field defects after normalisation of the intraocular pressure, Int Ophthalmol, 1989;13:113-17.

2. Michelson G, Groh MJ, Screening models for glaucoma, Curr Opin Ophthalmol, 2001:12:105-11.

3. Traverso CE, Walt JG, Kelly SP, et al., Direct cost of glaucoma and severity of the disease; a multinational long-term study of resource utilization in Europe, Br J Ophtha/mol, 2005;89;1245-9.

4. The Advanced Glaucoma Intervention Study (AGIS); The AGIS Investigators, The relationship between control of intraocular pressure and visual field deterioration, Am J Ophthalmol, 2000;130(4):429-40.

5. Sandhu SS, Chattopadhyay S, Birch MK, et al., Frequency of Goldmann applanation tonometer calibration error checks, J Glaucoma, 2005; 14(3):215-18.

6. Reus NJ, de Graaf M, Lemij HG, Accuracy of GDx VCC, HRT and clinical assessment of stereoscopic optic nerve head photographs for diagnosing glaucoma, Br J Ophthalmol, 2007;91(3):313-18.

7. Reus NJ, Lemij HG, European Optic Disc Assessment Trial (EODAT) group, Assessment of stereoscopic optic disc photographs in glaucoma by European ophthalmologists, Invest
Ophthalmol Vis Sci, 2007:48:E-Abstract 1970

8. Sommer A, Katz J, Quigley HA, et al., Clinically detectable nerve fiber atrophy precedes the onset of glaucomatous field loss, Arch Ophthal, 1991;109:77-83.

9. Colen TP, Lemij HG, Motion artifacts in scanning lase polarimetry, Opthalmology, 2002;109:1568-72.

10. Greenfield D, Knighton RW, Huang $X$, Effect of corneal polarization axis on assessment of retinal nerve fiber layer thickness by scanning laser polarimetry, Am I Ophthalmol, 2000;129:715-22.

11. Knighton RW, Huang $X$, Linear birefringence of the central human cornea, Invest Ophthalmol Vis Sci, 2002:43:82-6.

12. Weinreb RN, Bowd C, Zangwill LM, Glaucoma detection using scanning laser polarimetry with variable corneal polarization compensation, Arch Ophthalmol, 2003:121(2):218-24.

13. Medeiros FA, Zangwill LM, Bowd C, et al., Fourier analysis of scanning laser polarimetry measurements with variable corneal compensation in glaucoma, Invest Ophthalmol Vis Sci, 2003:44: 2606-12.

14. Shuman H, Murray J, DiLullo C, Confocal microscopy: an overview, Biotechniques, 1989;7:154-63.

15. Chauhan BC, McCormick TA, Nicolela MT, et al., Optic disc and visual field changes in a prospective longitudinal study of patients with glaucoma, Arch Ophthalmol, 2001;119:1492-9.

16. Huang D, Swanson EA, Lin CP, et al., Optical coherence tomography, Science, 1991;254(5035):1178-81.

17. Schuman JS, Hee MR, Arya AV, Optical coherence tomography: a new tool for glaucoma diagnosis, Curr Opin Ophthalmol, 1995;6(2):89-95.

18. Sihota R, Sony P, Gupta V, Diagnostic capability of optical coherence tomography in evaluating the degree of glaucomatous retinal nerve fibre damage, Invest Ophtha/mol Vis Sci, 2006;47(5):2006-10.

19. Schuman JS, Pedut-Kloizman T, Hertzmark E, et al., Reproducibility of nerve fiber layer thickness measurements using optical coherence tomography, Ophthalmology, 1996; 103(11):1889-98.

20. Medeiros FA, Zangwill LM, Bowd C, et al., Comparison of the GDx VCC scanning laser polarimeter, HRT II confocal scanning laser ophthalmoscope, and stratus OCT optical coherence tomograph for the detection of glaucoma, Arch Opth, 2004; 122:827-37

21. Deleón-Ortega JE, Arthur SN, McGwin G Jr, et al., Discrimination between glaucomatous and nonglaucomatous eyes using quantitative imaging devices and subjective optic nerve head assessment, Invest Opth Vis Sci, 2006;47:3374-81. 